\title{
A tribute to Beth Levine (1960-2020)
}

B cine and microbiology at UT Southwestern and investigator at the Howard Hughes Medical Institute, passed away on June 15, 2020, of metastatic breast cancer (Figure 1). She is survived by her husband, Dr. Milton Packer, a cardiologist and expert in heart failure, and her children, Rachel and Benjamin. Beth was renowned for her seminal work in autophagy, a pathway of intracellular macromolecule recycling that is conserved throughout eukaryotic organisms. In addition to cloning the first molecular component of this pathway in mammals, Beth demonstrated autophagy's role in processes from immunity to tumor suppression to aging, thereby placing it squarely within the realm of physiology. She was known for her incisive intellect, demand for scientific rigor, and commitment to mentoring. Beth's leadership in both clinical medicine and research made her a paragon of the physician-scientist during a time when it has become less common to cultivate personal excellence in both areas simultaneously. She was a member of the American Society for Clinical Investigation from 2000 and won its top honor, the Stanley J. Korsmeyer Award, in 2014.

Beth spent most of her life in the mid-Atlantic and northeastern United States. She was born in Newark, New Jersey, majored in French at Brown University, and earned her MD from Cornell University Medical College in 1986. Beth was an internal medicine resident at the Mount Sinai Hospital in New York and a fellow in infectious diseases at The Johns Hopkins Hospital. She began her independent career at Columbia University College of Physicians and Surgeons, where she rose to the rank of associate professor of medicine. In 2004, Beth was recruited to UT Southwestern as the Jay P. Sanford Professor in Infectious Diseases. She served as chief of infectious diseases until 2011, when she became the founding director of UT Southwestern's Center for Autophagy Research, which Beth built to

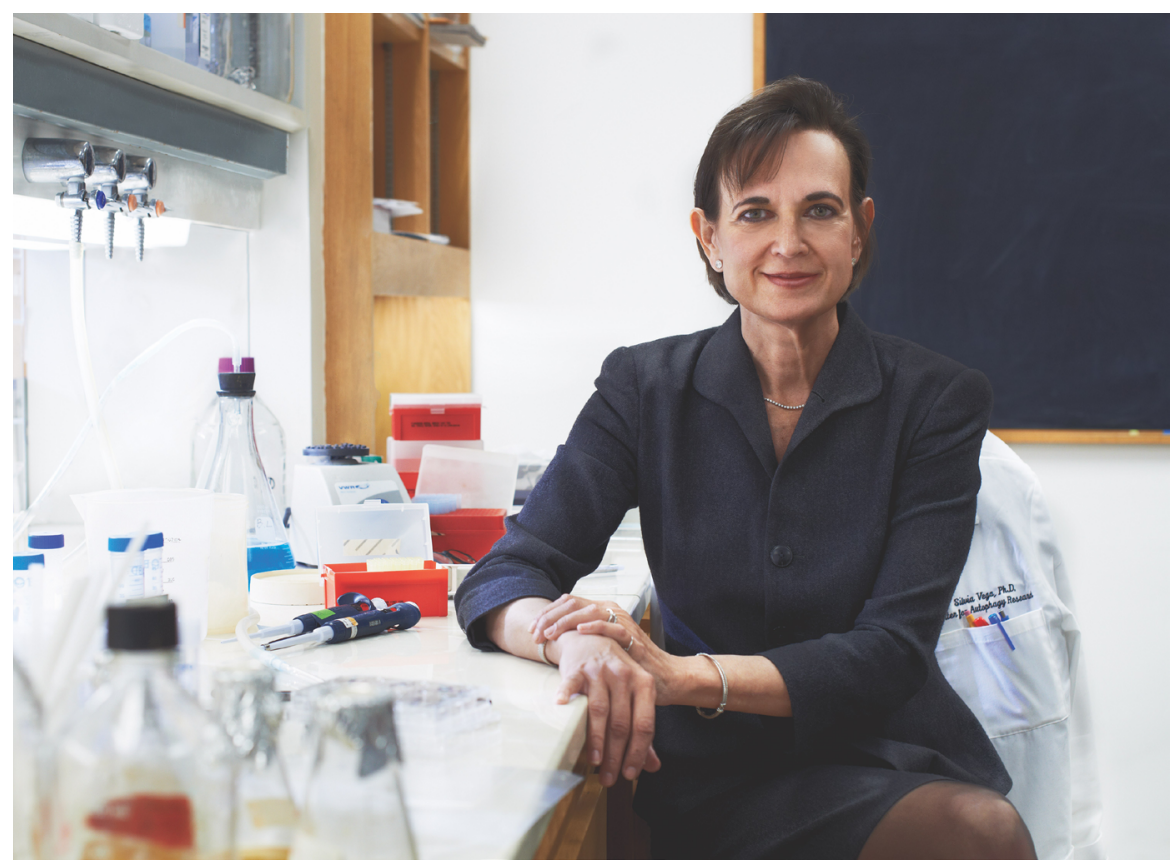

Figure 1. Beth C. Levine, MD. Image credit, Trevor Paulhus.

include seven faculty members and $23 \mathrm{sci}$ entists by 2020 .

Beth's landmark work in autophagy arose from an exploration into the mechanisms by which cells respond to viral infection, starting with her work in Marie Hardwick's lab at Johns Hopkins. Beth showed that infection with Sindbis virus induced apoptosis in cultured cells and brains of neonatal mice and that this response could be overcome by expressing the antiapoptotic protein $\mathrm{Bcl} 2(1,2)$. Later in her own lab, in an effort to better understand Bcl2-dependent neuroprotection, Beth used a yeast two-hybrid screen to identify Bcl2-interacting proteins. The screen produced just a single colony from one million transformants, but that clone launched a new direction of research for the Levine lab and the autophagy field. It contained the sequence of an unknown gene, now termed beclin-1 (BECN1), which encodes a coiled-coil protein widely expressed in mammalian tissues (3). Two critical insights about BECN1 quickly followed from the lab: first, that one allele of BECN1 is frequently deleted in some human cancers (4); and second, that BECN1 (by then recognized as being homologous to the yeast autophagy gene Apg6) induces autophagy and suppresses tumor formation in MCF7 human breast cancer cells (5). These findings not only described the first autophagy gene in mammals; they also established the first compelling link between autophagy and a human disease.

Prior to these discoveries, most autophagy research focused on yeast, in which Yoshinori Ohsumi, Daniel Klionsky, and others had defined the molecular components of the pathway and its role in nutrient stress responses, as explained by Levine and Klionsky in their commentary on Ohsumi's 2016 Nobel Prize in Physiology or Medicine (6). In this context, Beth's discoveries were pioneering in the truest sense of the word: they helped bring autophagy research into the new territory of mammalian physiology and disease. In addition to her original findings about Beclin-1's importance in tumor suppression and innate immunity against viral infections, Beth and her lab described many other contexts in which autopha- 
gy supports tissue function and homeostasis. These included embryogenesis, mitigation of age-related neurodegeneration, and the metabolic benefits of exercise, demonstrating again and again the health-promoting aspects of autophagy. Beth's work also suggested that manipulating autophagy in humans might produce therapeutic benefits in neurological and other diseases, an idea she pursued in part by cofounding the pharmaceutical company Casma Therapeutics.

Beth thought it was important to stimulate crosstalk among autophagy researchers and worked to make this happen, chairing the first Gordon Research Conference on Autophagy in Stress, Development and Disease in 2003 and serving as an organizer for the first Keystone Symposium on Autophagy in Health and Disease in 2007. In addition to demonstrating the rising profile of the field, these conferences had an amplifying effect on autophagy research, achieved by bringing together investigators from around the globe working in different organisms and on different problems related to cellular stress and disease. Beth was a catalytic factor in these interactions. She was exceptionally talented in crossdisciplinary science, capitalizing on observations from one field to understand processes in another. This skill helped her understand how defects in the molecular components of autophagy resulted in such complex phenotypes in mammals.

Beth became a Howard Hughes Medical Institute investigator in 2008 and won the Edith and Peter O'Donnell Award from The Academy of Medicine, Engineering \& Science of Texas the same year. She was elected to the Association of American Physicians in 2005 and the National Academy of Sciences in 2013. None of these accolades surprised those who knew Beth and followed her science. Beth was an exceptionally creative and courageous experimentalist with an uncompromising demand for rigor. Her lab's publications were mandatory reading in the autophagy field, and her lectures were famous for their clarity and precision. Beth's meticulous and disciplined nature extended to all areas of her professional life. In large international symposia and local committee meetings alike, she asked tough, incisive questions borne from curiosity and the desire to get to the bottom of the issue at hand.

Beth's colleagues and team saw her as someone with high standards, tough to impress, but who cared deeply about her people and advocated for them, trainees and staff alike. She was determined to make important discoveries, but nevertheless, her lab knew her as a patient and generous mentor with unwavering ethics. She went out of her way to help young investigators, even from outside her home institution, and she tried to connect researchers with common interests. Beth spent a great deal of time teaching trainees how to communicate their science, helping them identify and emphasize the most important aspects of their discoveries. She was a fantastic writing coach, working with her trainees throughout the process of preparing grants and manuscripts, not simply editing their drafts, but explaining the rationale behind each edit. Beth also loved to celebrate. New papers were recognized with a cake customized to reproduce a key piece of data in the icing. The entire group - the lab, clinical division, and Center for Autophagy Research - gathered at Beth and Milton's house for parties in the summer and winter, complete with a karaoke machine and lots of singing.

Beth was a particularly dedicated champion for physician-scientists and sought to inspire in them the same "joy of scientific curiosity and discovery," as she called it, that fueled her own career. She saw the value in bringing a clinically grounded perspective to bear on fundamental questions and tried to find people who could do this. Beth sought out budding scientists from the ranks of medical fellows at UT Southwestern and did as much as she could to cultivate their talent. In her interview in JCI after winning the 2014 Korsmeyer Award (7), Beth connected her decision to train in medicine to the humanistic appeal of helping individual patients. Her focus shifted to research when it became apparent that life as a scientist would allow her to solve the larger puzzles that produce genuine insights and long-term impact on disease. "I think what was most critical to my success was my willingness to follow my scientific intuition and curiosity and pursue questions that I thought were important," she said. What Beth found interesting and important over her career as an investigator left an indelible impact on the field of autophagy and on the countless scientists she inspired.

\section{Acknowledgments}

The author thanks Eileen White, Cindy Jozefiak, Richard Wang, Michael Shiloh, and Haley Smith for sharing their memories and insights.

\section{Ralph J. DeBerardinis}

Address correspondence to: Ralph J. DeBerardinis, 6000 Harry Hines Boulevard, Dallas, Texas, 75235, USA. Phone: 214.633.1804; Email: ralph.deberardinis@ utsouthwestern.edu.

1. Levine B, Goldman JE, Jiang HH, Griffin DE, Hardwick JM. Bc1-2 protects mice against fatal alphavirus encephalitis. Proc Natl Acad Sci USA. 1996;93(10):4810-4815.

2. Levine B, Huang Q, Isaacs JT, Reed JC, Griffin DE, Hardwick JM. Conversion of lytic to persistent alphavirus infection by the bcl-2 cellular oncogene. Nature. 1993;361(6414):739-742.

3. Liang XH, et al. Protection against fatal Sindbis virus encephalitis by beclin, a novel Bcl-2-interacting protein. J Virol. 1998;72(11):8586-8596.

4. Aita VM, et al. Cloning and genomic organization of beclin 1 , a candidate tumor suppressor gene on chromosome 17q21. Genomics. 1999;59(1):59-65.

5. Liang XH, et al. Induction of autophagy and inhibition of tumorigenesis by beclin 1 . Nature. 1999;402(6762):672-676.

6. Levine B, Klionsky DJ. Autophagy wins the 2016 Nobel Prize in Physiology or Medicine: Breakthroughs in baker's yeast fuel advances in biomedical research. Proc Natl Acad Sci USA. 2017;114(2):201-205.

7. Jackson S. Beth Levine receives the 2014 ASCI/ Stanley J. Korsmeyer Award. JClin Invest. 2014;124(4):1423-1424. 